SESSION 4. THEORETICAL SPECTROSCOPY 


\title{
DISTORTED WAVE CALCULATIONS: APPLICATION TO ASTROPHYSICS AND TOKAMYK PLASMA
}

\author{
A. K. Bhatia \\ Laboratory for Astronomy and Solar Physics \\ NASA/Goddard Space Flight Center \\ Greenbelt, Maryland 20771 USA
}

During the last few years, observations of solar phenomena have been carried out by rocket flights, manned satellites like Skylab, unmanned satellites like Orbiting Solar Observatories and more recently Solar Maximum Mission. The wavelengths, line intensities and line profiles in UV and $X$-ray regions of the solar spectra have been measured. The spectroscopic data obtained are of high spatial and spectral resolution. The goal is to understand the physical properties of the emitting plasma and determine the electron temperatures, densities and volume of the emitting plasma from UV and X-ray spectra.

Since emission lines are seen, this would imply plasma is hot and ionized. The lines are produced when the higher states of the positive lons excited by electron impact decay to the lower states. Therefore, it is necessary to understand the line forming processes and calculate the relevant atomic data for the interpretation of the spectroscopic data. Since the tokamak spectra are similar to the flare spectra, the same atomic data can be used to interpret the tokamak spectra. given by

The intensity of the line due to the transition from $j$ to $i$ is

$$
I_{j i}=N_{j} A_{j i} \frac{h c}{\lambda_{i j}},
$$

where $\lambda_{j j}$ is the wavelength of the line, $A_{j i}$ is the transition rate and $N_{j}$ is the population of the upper level. In the solar corona and in flares the electron densities are of the order of $10^{9}$ to $10^{12} \mathrm{~cm}^{-3}$ which are not high enough to maintain a Saha-Boltzmann distribution of level populations. These must be obtained by solving the statistical equilibrium equations.

We assume that the collisional and radiative processes are much faster than the ionization and recombination processes so that the calculation of the level populations can be carried out separately by inciuding only collisional and radiative processes. Therefore, we can deal with one ion at a time and assume that ionization equilibrium exists. This is a good approximation for the lower excited states but not for the higher excited states. We would al so assume that the plasma is optically thin.

The level populations $N_{i}$ are given by the rate equation

$$
\frac{d N_{i}}{d t}=-N_{e} N_{i} \sum_{j>i} C_{i j}^{e}+\sum_{j>i} N_{j} A_{j i}-N_{i} \sum_{j<i} A_{i j}+N_{2} \sum_{j>i} N_{j} C_{j i}^{\alpha},
$$


where $C_{i j}^{e}$ is the collisional excitation rate coefficient from level $i$ to $\mathrm{j}$ and is given by averaging over the Maxwellian distribution

$$
C_{i j}^{e}=\frac{8.63 \times 10^{-6}}{\omega_{i} k T_{e}^{3 / 2}} \int_{\Delta E_{i j}} \Omega_{i j} e^{-E / k T_{e}} d E \quad \mathrm{~cm}^{-3} \mathrm{~s}^{-1} \text {. }
$$

$T_{e}$ is the electron temperature. Collision strengths $\Omega_{i j}$, dimensionless and symmetric in $i$ and $j$, are related to the excitation cross section by

$$
\Omega_{i j}=E \omega_{i} \sigma_{i j} \quad,
$$

where $E$ is the incident energy and $\omega_{j}$ is the statistical weight of the lower level $i$. The de-excitation rate coefficient $c^{d} j$ is given by

$$
C_{j i}^{\alpha}=\frac{\omega_{i}}{\omega_{j}} C_{i j}^{e} e^{\Delta E_{i j} / k T_{e}} .
$$

Other processes which can populate or depopuiate the levels must be included in Eq. (2): photoexcitation by background radiation, as from the photosphere of the Sun, the rate for which is given by

$$
F_{i j}=D(h) A_{j i} /\left(e^{\Delta E_{i j} / k T_{R}}-1\right)
$$

where $T_{R}$ is the temperature of the blackbody radiation and $D(h)$ is the dilution factor. Stimulated emission, much smaller than spontaneous emission, and proton excitation rates? between the fine-structure levels should al so be included.

A number of approximations have been made to calculate $\Omega_{i j}$. The Coulomb Born approximation has been used extensively. This approximation overestimates collision strengths near the threshold for excitation. The Gaunt factor approximation based on the Bethe approximation, valid for allowed transitions, is not very reliable. The distorted wave approximation, which is valid when the coupling between the various channels is weak, has been widely used for the last few years. The total hamiltonian of the $(N+1)$ electron system is

$$
H_{N+1}=-\sum_{i=1}^{N+1}\left(\nabla_{i}^{2}+2 z / r_{i}\right)+\sum_{i \neq j}^{N+1} 2 / r_{i j},
$$

where $N$ is the number of target electrons and $Z$ the nuclear charge. The total wave function is

$$
\Psi_{n}=A \Phi_{n}(1,2, \ldots, N) F_{n}(N+1) \text {, }
$$

where $n$ is the initial or final state. $F_{n}$ satisfies the equation 


$$
\left(\frac{d^{2}}{d r^{2}}-\frac{l_{n}(\ln +1)}{r^{2}}+V_{n n}(r)+k_{n}^{2}\right) F_{n}(r)=0 .
$$

In the distorted wave programs ${ }^{3}$ developed at University College London (UCL) $V_{n n}$ is chosen to be a central potential and is given by a scaled Thomas-Fermi potential such that

$$
\operatorname{Lim}_{r \rightarrow \infty} V_{n n}(r)=2(Z-N) / r
$$

The functions $F_{n}(r)$ which have the usual limit for $r \rightarrow 0$ and $\infty$ are made orthogonal to the target orbitals having the same angular momentum. The reaction matrix is given by

$$
\begin{aligned}
K_{i j} & =-\left\langle\Psi_{i}\left|H_{N+1}-E_{T}\right| \Psi_{j}\right\rangle \\
& =-\left\langle F_{i}\left|V_{i j}+W_{i j}\right| F_{j}\right\rangle
\end{aligned}
$$

where $V_{j j}$ and $W_{i j}$ are the direct and exchange potentials. The short range correlations ${ }^{3}$ can be improved by including quadratically integrable functions in the wave functions (8). The reaction matrix $K_{i j}$ is modified then. The $T$ matrix is given by

$$
T=-\frac{2 i k}{1+i k}
$$

and the collision strangth for a given parity and total angular and spin momenta $L$ and $S$ is given by

$$
\Omega_{i j}^{\pi L S}=\frac{1}{2}(2 L+1)(2 S+1) \sum_{\ell_{i}, \ell_{j}}\left|T_{i j}\right|^{2} .
$$

The total collision strength is given by

$$
\Omega_{i j}=\sum_{\pi L S} \Omega_{i j}^{\pi L S}
$$

In general, only the first fow incident partial waves $\ell_{j}$ are used in the calculation. The contribution for $\ell>\ell_{i}$, for allowed transitions, is included in the Coulomb Bethe approximation 4 .

A better approxination is a close coupling approximation ${ }^{5}$. Though the calculations tend to be difficuit and expensive, they have the advantage of including resonances.

The target wave furctions $\Phi_{n}$ are calculated using the 'Superstructure' programb, also developed at UCL. The radial functions are again calculated in a scaled Thomas-Fermi Potential. As pointed out by Lazer that to obtain accurate wave function of highly ionized systems, it is necessary to include all configurations in the same complex of a given principal quantum number and parity. The configuration interaction type wave functions can be used in this program to calculate the energy levels, oscillator strengths and radiative transition rates in LS and intermediate couplings. The term 
coupling coefficients are calculated in this program to transform LS coupling collision strengths to collisions strengths in intermediate coupling 8 . Since we require atomic data for high nuclear charge $Z$, the relativistic corrections are calculated using the Breit-Pauli Hamiltonian as a perturbation to the non-relativistic Hamiltonian. Atomic data have been calculated for a number of ions, and the leve 1 populations and intensity ratios have been calculated for diagnostic purposes by solving the statistical equilibrium equations (2) by assuming that the steady state $\frac{d N_{i}}{d t}=0$ exists. We discuss some specific cases.

UV 1 ines of Ca XVII have been observed by Doschek el a ${ }^{9}$ in the August 9,1973 solar flare by using NRL's slitless objective grating (171-530Á) aboard Skylak. Also X-ray lines have been identified by McKenzie and Landecker ${ }^{10}$ in solar flares obtained from the Solex Bragg, crystal spectrometer aboard the USAF P78-1 satellite. Bhatia and Mason 11 calculated the atomif data using three sets of configurations: Case $A: 2 s^{2}, 2 s p, 2 p^{2}$; Case $B$ : Case $A+2 s 3 s$, $2 s 3 p, 2 s 3 d$; and Case C: Case $B+2 p 3 s, 2 p 3 p, 2 p 3 d$.

The energy values for Cases $B$ and $C$ are in good agreement with the observed values. The collision strengths are calculated using Case $B$ configurations and scaled. The collision strengths are in good agreement with the close coupling calculations of Dufton el al ${ }^{5}$.

The rates for electric dipole, electric quadrupole and magnetic dipole transitions are proportional to $Z^{4}, Z^{6}$ and $Z^{10}$, respectively. The rates for magnetic transitions at high $Z$ become comparable to rates for the allowed transitionsand therefore, the transitions become very important in the observations of tokamak plasma. The collision strengths for the dipole allowed, spin-forbidden and non-dipole allowed transitions behave $1 \mathrm{ike} \log E, 1 / \mathrm{E}^{2}$ and constant, respectively, as $E>\infty$. Aiso $z^{2} \Omega_{i j} \rightarrow$ constant for a fixed $E / Z^{2}$. These limits are usefut for interpolation or extrapolation of $A_{j i}$ and $\Omega_{i j}$.

The level populations and intensity ratios have been calculated for UV and $X$-ray 1 ines. The observed ${ }^{9}$ intensity ratio for the 1 ines 192.86 and $232.83 \AA$ corresponding to the transitions $2 \mathrm{~s} 2 \mathrm{p} \mathrm{p}_{1} \rightarrow$ $2 s^{2} 1_{\text {so }}$ and $2 p^{2}{ }^{3} \mathrm{P}_{2}>2 s 2 \mathrm{p}^{3} \mathrm{P}_{2}$ is 60 . The calculated ratios are $87.6,62.9$ and 59.1 for $\log \mathrm{Ne}=12,13$, and $14 \mathrm{~cm}^{-3}$, respectively. A comparison wjth the observed value of 60 would imply an electron density of $5 \times 10^{13} \mathrm{~cm}^{-3}$ which is much higher than $3 \mathrm{x}$ $10^{11} \mathrm{~cm}^{-3}$ at $6.3 \times 10^{6} \mathrm{~K}$ obtained by Dere et a $1^{12}$ from the measurements of $\mathrm{Ca} X V$ and $X V I$. This would imply an uncertainty in the calibration or the $233 \AA$ line $j$ s blended. In the $X$-ray region, the line at $20.456 \mathrm{~A}^{\circ}\left(2 \mathrm{~s} 3 \mathrm{~d}-\mathrm{O}_{3}>2 \mathrm{~s} 2 \mathrm{p} 3 \mathrm{P}_{2}\right)$ is density sensitive. But

the electron density was not determined as the measured intensities are weak. A line observed at $22.025 \AA$ can be associated with the calculated line $22.152 A^{\circ}\left(2 s 3 s\right.$ So $\left.\rightarrow 2 s 2 p 1 p_{1}\right)$. A similar calculation has been carried out for Fe XXIII by Bhatia and Mason ${ }^{3}$. Since then the identification of the lines in the $X$-ray region given by them has been 
confirmed by McKenzie and Landecker ${ }^{14}$. The lines at 263.76 and $132.83 \AA$ due to the transitions $2 \mathrm{~s} 2 \mathrm{p}^{3} \mathrm{P}_{1}>2 \mathrm{~s}^{2} 1_{\mathrm{S}_{0}}$ and $2 s 2 p 1_{P_{1}}>2 s^{2}{ }^{1} S_{Q_{R}}$ have been seen by Hinnor ${ }^{15}$ in PLT tokamak.

The line at $263.76 \AA$ obtained in NRL's spectroheliograph is an important 1 ine and has been used to study the energy release processes in solar flares. The most intense line of Fe XXIII in the UV region has been seen by Kastner et al ${ }^{I 5}$ using the GSFC grating spectrometer on 0SO-5.

The atomic data have been calculated for Mg VI, Si VIII, S X, Ar XII, etc by using the configurations $2 s^{2} 2 p^{3}, 2 s 2 p^{4}$ and $2 p^{5}$ by Feldman el a $1^{17}$. The 1 ine ratios $\left.\left({ }^{2} P_{3 / 2}>{ }^{4} S_{3 / 2}\right) /{ }^{2} D_{3 / 2} \rightarrow{ }^{4} S_{3 / 2}\right)$ and $\left({ }^{2} D_{3 / 2}>{ }^{4} s_{3 / 2}\right) /\left({ }^{2} D_{5 / 2}>{ }^{4} s_{3 / 2}\right)$ are density sensitive but not temperature sensitive. These transitions are within the levels of the ground configuration $2 s^{2} p^{3}$. S $x$ lines have been observed in quiet and active regions of the Sun using NRL's spectrograph aboard Skylab. Using the observed ratios for the lines 1213.00 and $1196.26 \AA$ corresponding to the transitions $2 s^{2} 2 p^{3}\left({ }^{2} D_{3 / 2}>{ }^{4} S_{3 / 2)}\right.$ and $2 s^{2} 2 p^{3}\left({ }^{2} D_{5 / 2} \rightarrow{ }^{4} s_{3 / 2}\right)$ respectjuely, Feldman et al 17 inferred an electron density of $10^{9} \mathrm{~cm}^{-3}$ at $1.3 \times 10^{6} \mathrm{~K}$ in quiet and active regions of the Sun. A similar calculation has been carried out for Fe XX. The line at $2665.1 \AA$ and $824.1 \AA$ corresponding to the transitions $2 s^{2} p^{3}\left({ }^{2} D_{5 / 2}>{ }^{2} D_{3 / 2}\right)$ and $2 s^{2} 2 p^{3}\left({ }^{2} D_{3 / 2}>{ }^{4} s_{3 / 2}\right)$ have been seen by Suckewer and Hinnov in PLT tokamak.

Doschek et a ${ }^{19}$ inferred the densities in the quiet sun and in coronal holes by comparing the intensities of the intersystem and allowed lines emitted by the ions formed in the transition zone. They used the lines at $1402.77 \AA\left(3 p{ }^{2} p_{1 / 2}>3 s^{2} S_{1 / 2}\right)$ in Si IV, $1908.73 \AA\left(2 s 2 p P_{1}>2 s^{2} 1_{S o}\right)$ in C III, and $1666.15 \AA$ $\left(2 s 2 p^{3} S_{2}>2 s^{2} p^{2}{ }^{3} P_{2}\right)$ in 0 III. But the intersystem and allowed lines were not close in wavelength. Therefore, when comparing the intensities of the various lines, it was necessary to take into account the change in temperature of the emitting plasna as well as the variation of the instrument reflectivity with wavelength. Those problems can be avoided by using the line ratios of the $S$ IV $1406 \AA$ intersystem 1 ine and the $1403 \AA$ resonance line $j$ in Si IV. These lines have been observed from Skylab. Bhatia et a ${ }^{20}$ calculated the atomic data for S IV using the $3 s^{2} 3 p, 3 s 3 p^{2}$ and $3 s 3 d^{2}$

configurations. But the density they inferred for December 17, and 21, 1973 and January 21, 1974 flares was four times that inferred from 0 IV measurements. Bhadra and Henry 21 , and Dufton and Kingston ${ }^{22}$ by

using a larger set of configurations calculated the collision strengths in close coupling approximation. The latter calculations include resonances as well. Dufton et a ${ }^{23}$ calculated the densities in the quiet sun, coronal holes, active region $B$ and active region $C$ and these are closer to the densities deduced by using other ions. 
The intensity ratio of the allowed lines is temperature sensitive and it can be shown that

$$
I_{j i / I_{k i}} \propto e^{-\left(\Delta E_{i j}-\Delta E_{i k}\right) / k T_{e}} \text {. }
$$

This ratio is sensitive to changes to the electron temperature Te if $\left(\Delta E_{i j}-\Delta E_{j k}\right) / k T_{e} \sim 1$. Feldman and Doschek 24 inferred the electron temperature $=$ ion temperature using $\mathrm{Mg}$ II allowed lines. The intensity ratio of the satellite line to the resonance line is a function of electron temperature and can be used for temperature diagnostics 25 .

The Bowen fluorescence mechanism takes place when the wave length of a transition coincides with the wavelength emitted by another ion. This radiation when absorbed will populate the upper levels preferentially and therefore will change the level populations and intensities of the emitted radjation. He II Lyman a (304 $\AA$ ) coincides with the 0 III $2 p^{2} 3 p_{2}>2 p 3 d P_{2}$ wave length. Bhatia et a ${ }^{26}$ calcylated the atomic data for 0 III by using $2 s^{2} 2 p^{2}$, $2 s 2 p^{3}, 2 p^{4}, 2 p 3 s, 2 p 3 p$ and $2 p 3 d$ configurations. The photoexcitation by $\mathrm{He}$ II was taken into account by increasing the electron excitation rate coefficient $C$ by $P C$ where $C$ is the rate coefficient for exciting the $2 p 3 d{ }^{3} p_{2}$ from $2 p^{2} 3 p_{2}$. The total excitation rate is, therefore, $K C$, where $K=1+P$ The level populations e.g. of $2 p 3 d 3 p_{2}$ and $2 p 3 p{ }^{3} s_{1}$, were found to be $K$ dependent. Choosing $K=1000$ to obtain agreement of the calculated intensity of $304 \AA$ line with the intensity observed by Behring et al 27 , they calculate the intensities of other lines and multiplets and compare with the observation.

The calculated intensities of the non-photosensitive multiplets $508,526,703$ and $835 \AA$ are in good agreement with the observations of Varanazza and Reeves ${ }^{28}$. Also, the intensities of multipiet components $508.18,703.85$, and $702.90 \AA$ agree very well with the observations of Behring et a ${ }^{27}$. The intensity of the multiplet $374 \AA$ is higher when photoexcitation is ingluded but is lower than the observed intensity given by Raymond ${ }^{29}$. Dere 30 has pointed out that this line is blended, and according to Raymond (private communication), the observed intensity should be lower than that given in Ref. 29 . Finally, the line at $644 \AA$ will not be observed at all if photoexcitation is not present and the calculated intensity in the presence of photoexcjtation agrees very well with the observation of Veranazza and Reeves ${ }^{28}$ (see Fig. 4 of Ref. 26 for more details). Furthermore, the observed multiplet $644 \AA$ is due to 0 III and not N II or 0 II as suggested by Veranazza and Reeves 28 . 
Though the bulk of the plasma is usually hydrogen or deuterium, other gases are added in controlled quantities for diagnostic purposes. Since the temperature in a tokamak is comparable to temperatures reached in solar flares, various stages of ionization of elements are present. Atomic data for various ions of interest have been calculated using UCL programs. The forbidden transitions, in particular magnetic dipole, are of special interest. These lines fall in the UV and EUV spectral regions and are due to transitions between the levels of the ground configurations $2 s^{2} \mathrm{p}^{\mathrm{k}}$ of these ions. These lines are well separated in wave lengths and line blending is much less severe than in the case of allowed lines at shorter wave lengths. These transitions can be well observed to determine the line profiles. Ion temperatures can be inferred by measuring the full width at half maximum. Ion concentration and electron density can be determined from the intensities of these lines. Suckewer et al 31 observed lines from titanium ions and compared the observed intensities with the synthetic spectrum calcylated by Bhatia et, al 32 using the configurations $2 s^{2}$, $2 s 2 p^{k+1}, 2 p^{k+2}$. Suckewer el al 31 observed the Ti XiV 2115.3, Ti $X V$ 2544.8, Ti XVII 3834.4 and $3371.5 \AA$ lines along with the allowed $T i$ $X I X 169 \AA$ iine and their intensity variation with time. The ion density can be calculated by noting that if the level population of the upper level does not vary with electron density, as for $2 s^{2} 2 p^{4} 3 p_{1}$ of Ti XV, (see Fig. 3 of Ref. 32), then

$$
I_{j i}=\frac{A_{j i}}{4 \pi} \int N_{j} d V=\frac{n_{j} A_{j i}}{4 \pi} \int N_{T} d V,
$$

where $n j=N j / N t$ is the level of population of the level $j$. From the measured $I_{j i}$ and the calculated $n_{j} A_{j i}$ as indicated in Fig. 3 of Ref. 31, they obtained an ion density of $2.6 \times 10^{10} \mathrm{~cm}^{-3}$.

More recentiy Stratton et $a 1^{1}$ measured the intensity ratios of various lines in Fe XVIII to XXII and compared with intensity ratios inferred from the DW calculations. They conclude (See Table 1 of Ref. 1) that in general the agreement is within 30 percent, the expected accuracy of the distorted wave calculations. 


\section{REFERENCES}

1. B.C. Stratton, H.W. Moss and M. Finkenthal, Ap. J. 279, L31 (1984).

2. S.0. Kastner and A.K. Bhatia, Astron. Astrophys, 71, 211 (1979).

3. W. Eissner and M. J. Seaton, J. Phys. B 5, 2187 (1972).

4. A. Burgess and V. B. Sheorey, J. Phys. B 1,2403 (1974).

5. P. L. Dufton, A. E. Kingston and N. S. Scott, J. Phys. B 16, 3053 (1983).

6. W. Eissner, M. Jones and H. Nussbaumer, Comp. Phys. Comm. 8, 270 (1974).

7. D. Layzer, Ann. Phys. 8, 271 (1972).

8. H. E. Saraph, Comp. Phys. Comm. 3, 256 (1972).

9. G. A. Doschek, U. Feldman and K. P. Dere, Astron. Astrophs. 60, L11 (1977).

10. T. L. Mckenzie and P. B. Landecker, Ap, J. 254, 309 (1982).

11. A. K. Bhatia and H. E. Mason, Astron. Astrophys. Suppl. Ser. 52 115 (1982).

12. K. P. Dere, H. E. Mason, K. G. Widing and A. K. Bhatia, Astron. Astrophys, Supp 1. Ser. 50, 341 (1979).

13. A. K. Bhatia and H. E. Mason, Astron. Astrophys. 103, 324 (1981).

14. D. L. McKenzie and P. B. Landecker, Ap. J. 254, 309 (1982).

15. E. Hinnov. Ap. J. Lett. 230, L197 (1980).

16. S. 0. Kastner, W. M. Neupert and M. Swartz, Ap. J. 191, 261 (1976).

17. U. Feldman, G. A. Doschek, J. T. Mariska, A. K. Bhatia and H.E. Mason, Ap. J. 226, 674 (1978).

18. S. Suckewer and E. Hinnov, Phys. Rev. Lett. 41, 756, (1978).

19. G. A. Doschek, U. Feldman, A. K. Bhatia and H. E. Mason, Ap. J. 226, 1129 (1978).

20. A. K. Bhatia, G. A. Doschek and U. Feldman, Astron. Astrophys. $86,32(1980)$.

21. K. Bhadra and R. J. W. Henry, Ap. J. 240, 368 (1980).

22. P. L. Dufton and A. E. Kingston, J. Phys. B 13, 4277 (1980).

23. P. L. Dufton, A. Hibbert, A. E. Kingston and G. A. Doschek, Ap. J. 257, 338 (1982).

24. U. Feldman and G. A. Doschek, Ap. J. 212, L147 (1977).

25. A. H. Gabriel Mon. Not, R. Astr. Soc. I45, 241 (1972).

26. A. K. Bhatia, S. 0. Kastner and W. E. Behring, Ap. J. 254, 887 (1982).

27. W. E. Behring, L. Cohen, U. Feldman and G. A. Doschek, Ap. J. 203, $521(1976)$.

28. J. E. Veranazza and E. M. Reeves, Ap. J. Suppl. 37, 485 (1978).

29. J. C. Raymond, Ap. J. 224, 259 (1978).

30. K. P. Dere, Solar Phys. 77, 79 (1982).

31. S. Suckewer, R. Fonck and E. Hinnov, Phys. Rev. A 21, 924 (1980).

32. A. K. Bhatia, U. Feldman, and G. A. Doschek, J. Appt.

Phys. 51, 1464 (1979). 